\title{
Comparative fault location algorithms for regional distribution network with distributed generator
}

\author{
Wang xiaoxue ${ }^{1, \text { a }}$, Li zhenxing ${ }^{1, b}$, Wu yun ${ }^{2}$, Zhang jinwei ${ }^{1}$, Fan yumeng ${ }^{1}$, Wang \\ liang $^{1}$ \\ ${ }^{1}$ College of Electrical Engineering and New Energy,China Three Gorges University,443002, Yichang \\ Hubei Province, China; \\ ${ }^{2}$ No.38 Huayan Street, Wujiagang District, 443002,Yichang,Hubei Province, China \\ a348126848@qq.com, 'blzx2007001@163.com
}

Keywords: distribution network, distributed generator, modified matrix algorithm, fault location

\begin{abstract}
Traditional matrix algorithm endures advantages of low requirements in FTU, high accuracy in Single-supply and radiation distribution network and so on. However, it will meet with erroneous judgment when it is used traditional matrix algorithm as long as it inserts with distributed generator. This article mainly focuses on analyzing the limitation of uses of implementation rational, which deals with the implementation of the matrix algorithm in the fault location algorithms in distribution network, applied in the distribution network with distributed generation. Then realizing a modified T-matrix algorithm based on these problems remained to be solved.
\end{abstract}

Introduction: This algorithm structures the information matrix of the sectionalized distribution network [1-4] based on the location information offered by FTU and progressively modifies it based on the multi-branched directional information, requires the associated node judgment matrix and lists describing matrix of network based on the data flow collected from the SCADA system [5-6]. Finally, adding the network matrix to fault matrix to get the fault judgment matrix and determine the fault section [7-8] based on the criteria. The article discusses its implementation rationale in practical application and tests the application and accuracy of the modified T matrix algorithm in combination with case studies to improve the reliability of the T matrix algorithm [9] in solving the problems of the high misjudgment in location fault sections in the distribution network with multi-distributed generator based on traditional algorithm.

\section{The fault location rationale in distribution network based on rectangle algorithm and analysis of its limitations}

The basis principle of the application in traditional matrix algorithm of fault location in distribution network is the to combine the utilization of fault information required from the FTU based on the foundation of graph theory with the topology of the distribution network to acquire the describing network matrix and fault information matrix to realize the location of the fault based on the results of the criteria after the calculation between matrixes.

The network information collected from the SCADA of the distribution network is the data foundation of the automatic processing of the fault location. When operating normally, it can combine with the undirected graph describing network topology to acquire the describing network matrix D based on real-time switch status and the branch current information.

$$
d_{i j}= \begin{cases}1 & \text { vertex } j \text { is the entrance of the feeder area } i \\ -1 & \text { vertex } j \text { is the outlet of the feeder area } i \\ 0 & \text { vertex } j \text { is notdirectlylinked the feeder area } i\end{cases}
$$

When distribution network goes wrong, one get the fault information matrix $G$ from the fault current information collected by each FTU. 


$$
g_{j}= \begin{cases}1 & \text { the failurecurrent flows from the vertex } j \\ 0 & \text { the failurecurrent fails to flow fromthe vertex } j\end{cases}
$$

To get the fault judgment matrix $P=D \times G$ by matrix calculation of describing network matrix $D$ and fault information matrix $G$. By searching the matrix information corresponded in branch one of the matrix $P$, it can be judged that the failure current only exists in upstream node in this branch, thus the failure occurs in this branch.

\section{The fault-locating theory in distributed network based on modified T matrix algorithm}

Traditional matrix algorithm can not be applied in multiple power networks because of its lack in branch orientation matrix judgment; this article comes up with a modified T matrix algorithm which mainly focuses on the definition of associated positive direction, which means the positive direction from bus bar to FTU. The fault associated node judgment matrix can be formed according to the direction of fault branch, and then the fault section can be formed based on the first step of the research of the fault associated node judgment matrix. Furthermore, fault diagnosis can be formed according to the calculation of the network description matrix and failure information matrix in the failure section, finally, the result of fault-locating can be implemented based on the fault branch formed by the research of the fault judgment matrix.

\section{a) The judgment of fault section}

When failure occurs in distribution network, firstly, fault information matrix can be structured by the directional information offered by FTU, among of which $F_{i i}$ is diagonal entry of the matrix. If $F_{i i}=1$, then the panel point is able to monitor the direction of the nodes and the value of the current; if $F_{i i}=0$, then the panel point is unable to monitor the direction of the node and the value of the current. Assumed that $Y$ is the number of the positive direction of the multiple branch node associated direction offered by TFU, if $Y=1$, then it can be judged that the failure occurs in the positive feeder of the monitoring point; if $Y \geq 2$, it can be judged that the failure occurs in the feeder which monitoring point where the maximum short-circuit current duplicated pointed to.

To determine the direction of the fault branch of the matrix $F_{i}$. If $F_{j j}=1$, then $F_{i j}=1$; If $F_{j j}=0$, then $F_{i(j+1)}=1$. If there exists no multiple branch nodes adjoined with $F_{j}$, then $F_{j}$ remains unchanged.

To get the fault information describing matrix $\mathrm{F}$ by partition disposing the distribution network system graph with multiple $\mathrm{DG}$ and $F=\operatorname{diag}\left[\begin{array}{lllll}J_{1} & J_{2} & \cdots & J_{n}\end{array}\right]_{n \times 1}$, namely, $F=\operatorname{diag}\left[\begin{array}{llll}1 & 1 & \cdots & 1\end{array}\right]_{n \times 1}$. Then to acquire the fault associated node judgment matrix $J$ by modifying the fault branch direction information of the multiple branch nodes. The expression of the matrix $J$ is:

$$
J=\left[\begin{array}{ccccccc}
1 & J_{12} & J_{13} & & & \\
J_{21} & 1 & J_{23} & & & \\
J_{31} & J_{32} & 1 & & & & \\
& & & \cdots & & & \\
& & & & \cdots & & \\
& & & & \cdots & J_{(n-1) n} \\
& & & & J_{n(n-1)} & 1
\end{array}\right]_{n \times n} \quad(1) \quad D=\left[\begin{array}{cccccc}
0 & 1 & 0 & 0 & 0 & 0 \\
0 & 0 & 1 & 0 & 0 & 0 \\
0 & 0 & 0 & 1 & 0 & 0 \\
0 & 0 & 0 & 0 & 1 & 0 \\
0 & 0 & 0 & 0 & 0 & 1 \\
0 & 0 & 0 & 0 & 0 & 0
\end{array}\right]
$$

The expression is applied to multiple panel points. If $J_{i j} \cap J_{j i}=1$, the fault point is between the multiple branch node $i$ and $j$; if $J_{i i}=1$ and other elements in the row of $J_{i i}$ are 0 , then the fault point is lied in the lower line of the multiple branch node $i$. The number of the bud in Fig. 1 is 
only one, namely, $J$.

\section{b) Modified network describing matrix}

Differed from the network describing matrix in the traditional matrix algorithm, it should be set with associated positive direction, namely, the positive direction where the PWRS power supply outflows. If the branch number between the phase node $i$ and $j$ on the positive feeder is 1 , then $D_{i j}=1$. On the contrary, if the branch number between the phase node $i$ and $j$ on the positive feeder is 0 , then $D_{i j}=0$. The network describing matrix $D$ can be acquired by above steps and the expression of the matrix $D$ can be acquired by Fig. 1 as follows:

\section{c) Modified fault information matrix}

When failure occurs, if the associated direction of the fault current detected by FTU is positive, the signal will be transmitted from the center of the monitoring control, then $G_{i i}=1$. Or, if the associated direction of the fault current detected by FTU is negative, the signal will not be transmitted from the monitoring center, then at the mean time, $G_{i i}=0$. The fault information matrix $G$ can be acquired by steps above, and the expression of the matrix $G$ when node $\mathrm{F}$ failures according to the Fig.1 is formula (3).

\section{d) Fault judgment matrix}

Supposing that $T=D+G$, to get the fault judgment matrix $T$, the expression of matrix is formula (4).

$$
G=\left[\begin{array}{cccccc}
1 & 0 & 0 & 0 & 0 & 0 \\
0 & 1 & 0 & 0 & 0 & 0 \\
0 & 0 & -1 & 0 & 0 & 0 \\
0 & 0 & 0 & -1 & 0 & 0 \\
0 & 0 & 0 & 0 & -1 & 0 \\
0 & 0 & 0 & 0 & 0 & -1
\end{array}\right]
$$

$$
T=D+G=\left[\begin{array}{cccccc}
1 & 1 & 0 & 0 & 0 & 0 \\
0 & 1 & 1 & 0 & 0 & 0 \\
0 & 0 & -1 & 1 & 0 & 0 \\
0 & 0 & 0 & -1 & 1 & 0 \\
0 & 0 & 0 & 0 & -1 & 1 \\
0 & 0 & 0 & 0 & 0 & -1
\end{array}\right]
$$

According to the formation process of matrix $\mathrm{T}$, if $T_{i i}=1$ and $T_{i j}=0$, then the failure occurs in the end of the feeder of the panel point $i$. If $T_{i i}=1$ and all the points of $T_{i j}=1$ is $T_{j j}=0$ or $T_{j j}=-1$, then the failure occurs between the panel point $i$ and $j$. Besides that, if $T_{i i}=-1$ and all the points of $T_{i j}=1$ is $T_{j j}=0$, it also indicates that the failure occurs between the node $i$ and $j$. According to (4), $T_{2-2}=1, T_{2-3}=1$ and $T_{3-3}=-1$. It indicates that failure occurs between FTU2 and FTU3, namely, the failure occurs in panel point $F$.

\section{Analysis of examples}

According to Fig.1, it sets up a distribution network system graph with four groups of DG, among of which, J1、J2、J3、J6、J8、J10 are multiple branch panel points. This article takes failure points F1 F2 as examples in Fig.1 to analyze by applying with modified T matrix algorithm. To take multiple branch panel points as graph nodes, direct-connect nodes as graph branches to acquire the zoning map of the distribution network system with multiple DG as follows: 


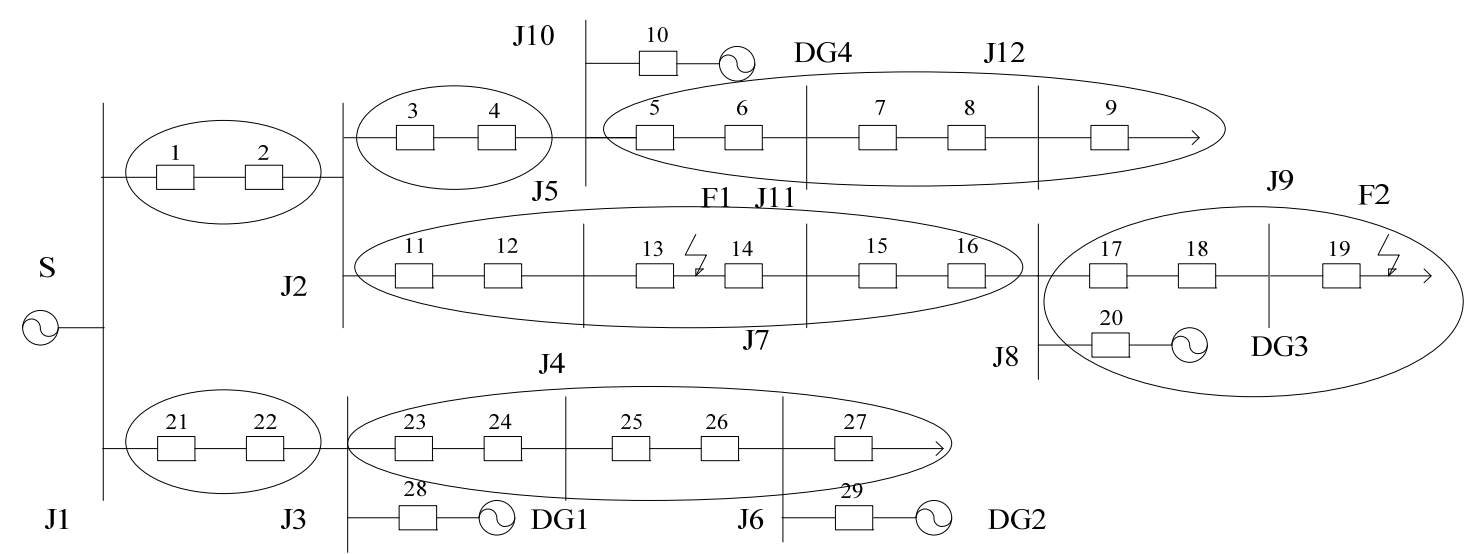

Fig.1 Figure of distribution network system with four groups of DG

\section{a) Matrix algorithm when failure occurs in point F1}

When failure occurs in F1, its information describing matrix is $F=\operatorname{diag}\left[\begin{array}{llllll}J 1 & J 2 & J 3 & J 6 & J 8 & J 10\end{array}\right]$, namely, $F=\operatorname{diag}\left[\begin{array}{llllll}1 & 1 & 1 & 1 & 1 & 1\end{array}\right]$. As long as the failure occurs, it can modify the multiple branch node fault branch direction information to acquire the fault associated node judgment matrix $J$ and $J$ is formula (5).

$$
J=\left[\begin{array}{llllll}
1 & 1 & 0 & 0 & 0 & 0 \\
0 & 1 & 0 & 0 & 1 & 0 \\
1 & 0 & 1 & 0 & 0 & 0 \\
0 & 0 & 1 & 1 & 0 & 0 \\
0 & 1 & 0 & 0 & 1 & 0 \\
0 & 1 & 0 & 0 & 0 & 1
\end{array}\right]
$$

$$
D=\left[\begin{array}{llllll}
0 & 1 & 0 & 0 & 0 & 0 \\
0 & 0 & 1 & 0 & 0 & 0 \\
0 & 0 & 0 & 1 & 0 & 0 \\
0 & 0 & 0 & 0 & 1 & 0 \\
0 & 0 & 0 & 0 & 0 & 1 \\
0 & 0 & 0 & 0 & 0 & 0
\end{array}\right]
$$

To accumulate $J_{i j}$ and $J_{j i}$ separately, according to the result, only $J_{28} \cap J_{82}=1$. Thus, it can be judged that the failure occurs between the multiple panel points $J_{2}$ and $J_{8}$. Moreover, to form the net work describing matrix with line switches $(11,12,13,14,15,16)$ between $J_{2}$ and $J_{8}$ to acquire the matrix $D$ and $D$ is formula (6).

It can be seen from the model diagram of the distribution network built up, when failure occurs, the failure current flowed from FTU situated in $11,12 、 13$ is the sum of the current from the system power-supply S and currents from power supply DG1、DG2、DG4, the positive direction of which is the associated positive direction set by the network. The currents situated in $14,15,16$ of the FTU is the failure currents offered by DG3 whose direction is negative to the associated positive direction set by the network. To acquire the fault information matrix from the overcurrent information from the FTU transmitted to SCADA and EMS, the matrix G is formula (7).

$$
G=\left[\begin{array}{cccccc}
1 & 0 & 0 & 0 & 0 & 0 \\
0 & 1 & 0 & 0 & 0 & 0 \\
0 & 0 & 1 & 0 & 0 & 0 \\
0 & 0 & 0 & -1 & 0 & 0 \\
0 & 0 & 0 & 0 & -1 & 0 \\
0 & 0 & 0 & 0 & 0 & -1
\end{array}\right]
$$

$$
T=\left[\begin{array}{cccccc}
1 & 1 & 0 & 0 & 0 & 0 \\
0 & 1 & 1 & 0 & 0 & 0 \\
0 & 0 & 1 & 1 & 0 & 0 \\
0 & 0 & 0 & -1 & 1 & 0 \\
0 & 0 & 0 & 0 & -1 & 1 \\
0 & 0 & 0 & 0 & 0 & -1
\end{array}\right]
$$

In turn, the sum of the network describing matrix and fault information matrix is formula (8).

It can be seen from the fault judgment matrix that $T_{13-13}=1, T_{13-14}=1$ and $T_{14-14}=-1$, which indicates that the failure occurs between switch 13 and 14, namely, the failure occurs in F1. 


\section{b) The matrix operation when failure occurs in F2}

When failure occurs in F2, the fault information describing matrix can be acquired $F=\operatorname{diag}\left[\begin{array}{llllll}1 & 1 & 1 & 1 & 1 & 1\end{array}\right]$. As long as the failure occurs, the fault associated node judgment matrix $J$ can be acquired, and $J$ is formula (9).

$$
J=\left[\begin{array}{llllll}
1 & 1 & 0 & 0 & 0 & 0 \\
0 & 1 & 0 & 0 & 1 & 0 \\
1 & 0 & 1 & 0 & 0 & 0 \\
0 & 0 & 1 & 1 & 0 & 0 \\
0 & 0 & 0 & 0 & 1 & 0 \\
0 & 1 & 0 & 0 & 0 & 1
\end{array}\right]
$$

$$
D=\left[\begin{array}{lllllllll}
0 & 1 & 0 & 0 & 0 & 0 & 0 & 0 & 0 \\
0 & 0 & 1 & 0 & 0 & 0 & 0 & 0 & 0 \\
0 & 0 & 0 & 1 & 0 & 0 & 0 & 0 & 0 \\
0 & 0 & 0 & 0 & 1 & 0 & 0 & 0 & 0 \\
0 & 0 & 0 & 0 & 0 & 1 & 0 & 0 & 0 \\
0 & 0 & 0 & 0 & 0 & 0 & 1 & 0 & 0 \\
0 & 0 & 0 & 0 & 0 & 0 & 0 & 1 & 0 \\
0 & 0 & 0 & 0 & 0 & 0 & 0 & 0 & 1 \\
0 & 0 & 0 & 0 & 0 & 0 & 0 & 0 & 0
\end{array}\right]
$$

To sum $J_{i j}$ and $J_{j i}$ separately, the result indicates that only $J_{88}=1$ and $J_{8 j}=0$. In this case, it can be judged that the failure occurs in the lower feeder of the multiple branch panel point $J_{8}$. In turn, to form the network describing matrix by line switches $(11,12,13,14,15,16,17,18,19)$ between $J_{2}$ and $J_{8}$ to acquire the matrix $D$ and $D$ is formula (10).

It can be seen from the model diagram of the distribution network system, when failure occurs, the failure current from the FTU situated at $11,12 、 13 、 14,15,16 、 17,18,19$ is the sum current from the system power supply S and current from generators DG1、DG2、DG3、DG4, whose positive direction is the associated positive direction set by the network. The fault information matrix can be acquired from the overcurrent information from FTU transmitted to SCADA and EMS, and $G$ is formula (11).

$$
G=\left[\begin{array}{lllllllll}
1 & 0 & 0 & 0 & 0 & 0 & 0 & 0 & 0 \\
0 & 1 & 0 & 0 & 0 & 0 & 0 & 0 & 0 \\
0 & 0 & 1 & 0 & 0 & 0 & 0 & 0 & 0 \\
0 & 0 & 0 & 1 & 0 & 0 & 0 & 0 & 0 \\
0 & 0 & 0 & 0 & 1 & 0 & 0 & 0 & 0 \\
0 & 0 & 0 & 0 & 0 & 1 & 0 & 0 & 0 \\
0 & 0 & 0 & 0 & 0 & 0 & 1 & 0 & 0 \\
0 & 0 & 0 & 0 & 0 & 0 & 0 & 1 & 0 \\
0 & 0 & 0 & 0 & 0 & 0 & 0 & 0 & 1
\end{array}\right]
$$$$
T=\left[\begin{array}{lllllllll}
1 & 1 & 0 & 0 & 0 & 0 & 0 & 0 & 0 \\
0 & 1 & 1 & 0 & 0 & 0 & 0 & 0 & 0 \\
0 & 0 & 1 & 1 & 0 & 0 & 0 & 0 & 0 \\
0 & 0 & 0 & 1 & 1 & 0 & 0 & 0 & 0 \\
0 & 0 & 0 & 0 & 1 & 1 & 0 & 0 & 0 \\
0 & 0 & 0 & 0 & 0 & 1 & 1 & 0 & 0 \\
0 & 0 & 0 & 0 & 0 & 0 & 1 & 1 & 0 \\
0 & 0 & 0 & 0 & 0 & 0 & 0 & 1 & 1 \\
0 & 0 & 0 & 0 & 0 & 0 & 0 & 0 & 1
\end{array}\right]
$$

In turn, the sum of the network describing matrix and fault information matrix is formula (12).

It can be seen from the failure judgment matrix that $T_{19-19}=1$, which indicates that the failure occurs at the end of feeder of the switch 19, namely, the failure occurs at F2.

\section{c) The implementation process}

The modified T matrix algorithm which the article comes up with combines the data current from FTU to SCADA with data current which EMS transmitted to accomplish the matrix algorithm and come into a snap judgment with the fault section of the distribution network. From the modified $\mathrm{T}$ matrix algorithm summarized above, the implementation process of the algorithm is:

(1) To assume a positive direction, namely, the positive direction is the direction from feeder to FTU;

(2) To from the fault associated dote judgment matrix $J$ from the orientation of the fault 
branch;

(3) To transmit the data from FTU to SCADA and EMS to form network describing matrix $D$;

(4) To form the fault information matrix $G$ from the fault information monitored by FTU;

(5) To sum the network describing matrix $D$ with fault information matrix $G$, namely, $T=D+G$

(6) To locate the fault section by the criterion.

\section{Summary}

By comparing and analyzing the criterion results of the former and latter modified matrix algorithm, it indicates that the modified $\mathrm{T}$ matrix algorithm solve the problem which the traditional matrix algorithm has in high false positive in distribution network with multiple DG. It greatly improve the accuracy in fault-locating in distribution network and enhance the stability and security in the operation of the power grid under a relatively low cost circumstance. In the mean time, it also fully endures an advantage of environmental protection of the distributed power supply.

\section{Acknowledgements}

This work was supported by the Hubei Province Natural Science Foundation of China (No. 2014CFB680).

\section{References}

[1] Hedayat Saboori, Reza Hemmati, Vahid Abbasi: Energy Conversion and Management, Volume 105, 15 November 2015, Pages 938-945

[2]Fathollah Bistouni, Mohsen Jahanshahi: Reliability Engineering \& System Safety, Volume 144, December 2015, Pages 128-146

[3]Reza Hemmati, Rahmat-Allah Hooshmand, Nabi Taheri: International Journal of Electrical Power \& Energy Systems, Volume 73, December 2015, Pages 665-673

[4]Byeong-Yeon Kim, Kwang-Kyo Oh, Hyo-Sung Ahn: Control Engineering Practice, Volume 43, October 2015, Pages 21-38

[5] B.R. Mehta and Y.J. Reddy: Chapter 7 - SCADA systems, In Industrial Process Automation Systems, edited by B.R. MehtaY.J. Reddy, Butterworth-Heinemann, Oxford, 2015, Pages 237-300

[6]Lucian Ioan Dulău, Mihail Abrudean, Dorin Bică: Procedia Technology, Volume 19, 2015, Pages 665-672

[7] Anamika Yadav, Aleena Swetapadma: International Journal of Electrical Power \& Energy Systems, Volume 69, July 2015, Pages 27-33

[8] Fábio Bertequini Leão, Rodrigo A.F. Pereira, José R.S. Mantovani: International Journal of Electrical Power \& Energy Systems, Volume 63, December 2014, Pages 787-805

[9] Joseph B. Bernstein, Moti Gabbay, Ofir Delly: Microelectronics Reliability, Volume 54, Issue 12, December 2014, Pages 2951-2955 